\title{
Anesthetic considerations in lung transplantation: past, present and future
}

\author{
Andrew W. Murray ${ }^{1}$, Michael L. Boisen ${ }^{2}$, Ashley Fritz ${ }^{3}$, J. Ross Renew ${ }^{4}$, Archer Kilbourne Martin ${ }^{3}$ \\ ${ }^{1}$ Department of Anesthesiology, Mayo Clinic Graduate School of Medicine, Mayo Clinic, Phoenix, AZ, USA; ${ }^{2}$ Department of Anesthesiology \\ and Perioperative Medicine, University of Pittsburgh School of Medicine, Pittsburgh, PA, USA; ${ }^{3}$ Division of Cardiothoracic and Thoracic \\ Anesthesiology, Mayo Clinic, Jacksonville, FL, USA; ${ }^{4}$ Department of Anesthesiology, Mayo Clinic Graduate School of Medicine, Mayo Clinic, \\ Jacksonville, FL, USA; ${ }^{5}$ Division of Cardiovascular and Thoracic Anesthesiology, Mayo Clinic, Jacksonville, FL, USA \\ Contributions: (I) Conception and design: AW Murray; (II) Administrative support: None; (III) Provision of study materials or patients: None; (IV) \\ Collection and assembly of data: None; (V) Data analysis and interpretation: None; (VI) Manuscript writing: All authors; (VII) Final approval of \\ manuscript: All authors. \\ Correspondence to: Andrew W. Murray. Department of Anesthesiology, Mayo Clinic Graduate School of Medicine, Mayo Clinic, Phoenix, AZ, USA. \\ Email: Murray.Andrew@mayo.edu.
}

\begin{abstract}
Lung transplantation is a very complex surgical procedure with many implications for the anesthetic care of these patients. Comprehensive preoperative evaluation is an important component of the transplant evaluation as it informs many of the decisions made perioperatively to manage these complex patients effectively and appropriately. These decisions may involve pre-emptive actions like pre-habilitation and nutrition optimization of these patients before they arrive for their transplant procedure. Appropriate airway and ventilation management of these patients needs to be performed in a manner that provides an optimal operating conditions and protection from ventilatory injury of these fragile post-transplant lungs. Pain management can be challenging and should be managed in a multi-modal fashion with or without the use of an epidural catheter while recognizing the risk of neuraxial technique in patients who will possibly be systemically anticoagulated. Complex monitoring is required for these patients involving both invasive and non-invasive including the use of transesophageal echocardiography (TEE) and continuous cardiac output monitoring. Management of the patient's hemodynamics can be challenging and involves managing the systemic and pulmonary vascular systems. Some patients may require extra-corporeal lung support as a planned part of the procedure or as a rescue technique and centers need to be proficient in instituting and managing this sophisticated method of hemodynamic support.
\end{abstract}

Keywords: Pulmonary hypertension (PHTN); frailty; protective lung ventilation; transesophageal echocardiography (TEE); cystic fibrosis (CF); extra-corporeal life support; regional pain management techniques

Submitted Jul 23, 2020. Accepted for publication Feb 10, 2021.

doi: $10.21037 /$ jtd-2021-10

View this article at: http://dx.doi.org/10.21037/jtd-2021-10

\section{Introduction}

Lung transplantation represents the ultimate therapy for patients with end-stage lung disease (1). In many ways it can be one of the most challenging surgical procedures to perform. In the same way the anesthetic care for these patients is extremely complex, multi-faceted and challenging.
From the time of the first lung transplant in 1963 by Dr. James Hardy and the first successful lung transplant with long term survival in 1983 by Dr. Joel Cooper, careful meticulous and well-coordinated anesthetic care has had to support the surgeon in order to provide this life-saving procedure to many patients.

Advances in airway management have assisted in these endeavors as has the development of ventilation 
strategies, inotropic and vasopressor regimens to support the hemodynamic condition of the patient. Efforts to control pain and the untoward effects thereof have also accompanied the steady initial rise of lung transplants.

Complex monitoring methods have had to be adopted in the form of invasive systemic and pulmonary pressure monitoring supplemented by intra-operative cardiac ultrasound with real-time interpretation has necessitated the development of finely-honed skills on the part of the anesthesiologist.

The development of immune suppression and modulation has led to better outcomes leading often to the need of these patients to return to the operating room for other nontransplant related surgical procedures. As such it is important, not only for the cardiac specialists, for the regular general anesthesiologists to understand some of the challenges these patients present and how best to manage them.

\section{Preoperative considerations}

For carefully selected patients with end-stage lung disease, lung transplantation offers improved survival and quality of life. Candidate selection and disease-specific criteria for timing of referral and listing are detailed in ISHLT guidelines (2-4) and summarized in a recent review in this journal (5). A multidisciplinary evaluation including anesthesia and intensive care physicians can identify concerns relevant to the conduct of the anesthetic, identify modifiable risks, and implement optimization strategies aimed at reducing complications throughout the perioperative period.

\section{Respiratory assessment}

Review of the patient's respiratory diagnoses, current management, and recent clinical status including functional capacity and oxygen requirements is key since patient condition may have deteriorated since listing. Recipient infection and colonization with resistant organisms must be accounted for in perioperative antibiotic coverage. Computed tomography of the chest provides high resolution data on lung anatomic abnormalities and should be reviewed for features impacting lung isolation technique (diameter of trachea and main bronchi, anomalous lobar bronchus e.g., bronchus suis). Spirometry with lung volumes provides important functional information on severity and type of ventilatory defect (restriction or obstruction), and arterial blood gas and DLCO values provide insight into gas exchange abnormalities. Ventilation perfusion scintigraphy provides quantitation of lung perfusion useful in determining which lung to transplant in a single lung transplant, or which lung to transplant first in a bilateral transplant (6).

\section{Cardiovascular disease}

Coronary artery disease (CAD) without end-organ ischemia or dysfunction is considered a relative contraindication to lung transplantation. Moderate $\mathrm{CAD}$ is present in in up to $20-30 \%$ of lung transplant candidates over age 50 , but does not appear to increase mortality $(7,8)$. Because history and noninvasive testing may be unreliable in end-stage lung disease patients, routine coronary angiography is common at many centers.

Lung transplant recipients with severe CAD amenable to revascularization appear to have acceptable outcomes (9).

Atrial fibrillation prior to transplant is associated with post-transplant arrhythmias and longer hospital stay (10). Presently there is limited evidence to support specific pharmacoprophylactic strategies for post-lung transplant AF. Known risk factors include male sex, dilated left atrium and double lung transplant.

Left ventricular diastolic dysfunction is an independent predictor of PGD, probably related to elevated pulmonary venous pressure exacerbating capillary leak of ischemiareperfusion injury (11).

\section{Pulmonary hypertension (PHTN)}

Both primary and secondary PHTN complicate transplant management and increase PGD risk in a dose-dependent fashion (odds ratio of 1.3 per $10 \mathrm{mmHg}$ mean pressure increase; 95\% CI, 1.1-1.5; $\mathrm{P}<0.001)(12)$. Clinical signs of right ventricular failure (drowsiness, pallor, cyanosis, ascites, edema, venous congestion) must be identified and hemodynamic support initiated to avoid decompensation on induction of anesthesia and initiation of mechanical ventilation. Absent signs of overt failure, echocardiographic findings of RV dilation, hypokinesis, and severe tricuspid regurgitation should prompt advanced monitoring, initiation of inotropic and RV afterload lowering therapies, and consideration of early planned extracorporeal support (13). Due to risk of PGD, some centers maintain veno-arterial extracorporeal membrane oxygenation (VA-ECMO) postoperatively for controlled postoperative graft reperfusion and allowing protective ventilation (14). 


\section{Renal dysfunction}

Kidney dysfunction is prevalent and a key determinant of post-transplant survival. Acute kidney injury affects $>50 \%$ of recipients and is severe enough to require renal replacement in $6 \%$. Perioperative etiologies include hypotension, decreased cardiac output, hypoxemia, and nephrotoxic medications (antibiotics and calcineurin inhibitors), often in combination. Risk factors for acute kidney injury include chronic kidney disease, $\mathrm{BMI}>30$, diabetes, ethnicity, and preoperative ICU or ECMO (15).

\section{Functional status and prehabilitation}

Functional status is an established prognostic indicator $(16,17)$. Six-minute walking distance $(6 M W D)$ is widely used and robustly predicts outcomes (18) to the extent that is one of the variables included in the LAS calculation. Supervised exercise training programs such as pulmonary rehabilitation have well established benefits for COPD and other diagnoses, and have been shown to improve exercise capacity and quality of life both pre- and posttransplant (19). A recent systematic review confirms that pre-transplant pulmonary rehabilitation improves 6-minute walking distance and patient-reported quality of life (20). A recent observational cohort study reported significant improvements in $6 \mathrm{MWD}$ and dyspnea scores in a cohort of 39 lung transplant candidates who were enrolled in pulmonary rehabilitation for as little as 3 weeks (21). Pulmonary rehabilitation programs are also an ideal forum for patient educational interventions (22).

Frailty, a syndrome of decreased physiologic reserve, is prevalent in the cardiothoracic transplant population (23). Two frailty screening instruments studied In the lung transplant population, the Fried Frailty Phenotype (FFP) and the Short Physical Performance Battery (SPPB), have been shown to predict disability and death or delisting before transplant (24). In a single center retrospective cohort, pretransplant FFP frailty was not associated with excess posttransplant mortality or ICU stay (25). In fact, frail patients experienced greater improvement with transplant, suggesting reversibility of lung disease-related frailty versus age-related frailty. However, in a larger multicenter cohort, SPPB (a 3 -part assessment of gait speed, chair stands, and balance) was an independent predictor of 1- and 4-year survival posttransplant (26). Further research is needed to validate frailty screening instruments, elucidate modifiable components, and define structured interventions to decrease risk.

\section{Nutrition, sarcopenia and obesity}

Malnutrition commonly accompanies end stage lung disease and portends poor outcomes. Underweight (BMI $<18.5)$ is associated with increased mortality $(27,28)$. Recent work showed that recipients at increased risk of death and major complications can be identified by a prognostic nutrition index calculated based on serum albumin level and peripheral blood lymphocyte count (29). Interventions to optimize nutrition include evaluation by a clinical dietician, oral nutrition supplementation, and enteral feeding tube placement. Perioperative immune-nutrition with arginine and omega-3 fatty acids has shown promise in reducing complications in other types of major surgery, but research is needed in the lung transplant population.

Decreased muscle mass and impaired muscle strength (sarcopenia) is also common in patients with end stage lung disease (25). In a cohort of 36 lung transplant recipients, a muscle index $<25^{\text {th }}$ percentile measured by CT measurement was independently associated with mortality (hazard ratio 3.83; 95\% CI, 1.42-10.3; P=0.007) and longer hospital length of stay (30). Muscle mass can also be assessed using dual X-ray absorptiometry (DXA), bioimpedance, ultrasound, and skin fold measurements. Muscle strength can be measured in the clinic by handgrip strength, quadriceps extension, and chair stands.

Class II-III obesity $(\mathrm{BMI}>35)$ is an absolute contraindication to lung transplant, conferring a nearly twofold increased risk of death (31). Obese patients may be able to lower risk by reducing BMI prior to transplant (32). Class I obesity (BMI 30-34.9) is currently considered a relative contraindication based on an association with mortality (28) and PGD (27), but similar adjusted survival compared to normal and overweight subjects was observed in a multi-center analysis (31). Interestingly, a subgroup of the same study found that circulating levels of leptin, a cytokine secreted by adipose tissue, predicted mortalitya finding corroborated in studies of ALI/ARDS in the non-transplant setting. Recently published results of the Lung Transplant Body Composition Study using CTbased adiposity quantification performed on clinically indicated chest CTs plus a single abdominal slice showed that subcutaneous but not visceral adipose is associated with increased plasma biomarkers and risk of PGD (33).

\section{Airway management}

The airway management of the early double lung transplants 
was initially single lumen intubation of the trachea as early procedures involved en-bloc transplantation on cardiopulmonary bypass $(\mathrm{CPB})$ with anastomosis of the airway occurring at the level of the trachea.

Evidence that tracheal anastomosis was more prone to dehiscence prompted the surgical approach for double lung transplantation, much like that of single lung transplantation, to be changed to bronchial anastomosis. This technique developed in the early 1990s to avoid the complications of bronchial and tracheal anastomosis disruption, the need for cardiac surgery and the use of CBP required for en-bloc transplantation.

This necessitated a change in airway management by the anesthesiologist to allow selective ventilation if the non-operative lung. The left sided DLEBT is favored due to the low degree of variation in left sided anatomy, the relatively longer left main bronchus as well as the potential of malalignment of the right-sided DLEBT. The use of the DLEBT also allowed the development of sequential single lung transplants to be performed with or without the use of any extra-corporeal circulation.

Another method of achieving lung isolation would be to make use of bronchial blockers (34). Whereas originally Fogarty vascular occlusion catheters were used for this purpose, the development of modern purpose-built bronchial blockers can allow for precise positioning of the blocker in the intended location under fiberoptic guidance. The pitfalls with these devices are that they can be prone to dislodgement and inability to apply effective suction to the distal airways is limited due to small diameter, but they can provide an isolation technique when the airway would be prohibitively challenging to place a DLEBT (34) as well as lessening the number of airway manipulations, all of which provide another risk for infection. The development of the EZ-blocker by Fuji also allowed placement to be relatively easy in addition to the ability to use the same blocker for both sides without the need to change position.

Often the lung transplant patient has very poor respiratory reserve and this would necessitate optimization of preoxygenation as well as a rapid-sequence induction to facilitate a prompt securing of the airway. In some cases, it is necessary to achieve this with a single lumen endotracheal tube (SLETT) and then exchanging to a DLEBT. Initial passage of the SLETT also provides the anesthesiologist to perform careful but aggressive pulmonary toilet in the recipients with suppurative lung disease.

\section{Ventilation management}

This has evolved from early transplants having patient manually ventilated (35) to the development of simple mechanical ventilation (36) to the advanced ventilators that we now have available. The accumulated knowledge that has been developed in the anesthetic and critical care literature relating to ventilator management, one lung ventilation (OLV) and management of acute respiratory distress syndrome (ARDS) have served to improve ventilator care of the lung transplant patient. ARDS can be approximated to primary graft dysfunction (PGD) that occurs to varying degrees in the lung transplant recipient. Much of the ventilator management has been extrapolated from the lessons learned from managing ARDS patients (36).

Lung transplant patients occupy a spectrum from lungs with low compliance in idiopathic pulmonary fibrosis (IPF) all the way to extremely high compliance in conditions like severe chronic obstructive pulmonary disease (COPD). This necessitates a careful ventilation strategy developed for each patient to provide adequate oxygenation and $\mathrm{CO}_{2}$ clearance while not causing deterioration of the hemodynamics or causing barotrauma.

The COPD patients will require low ventilating pressures $\left(\mathrm{P}_{\text {aw }}\right)$ with lower respiratory rate (RR), adequate expiration time with inspiratory to expiratory (I:E) ratio of 1:3 or even 1:4. These patients are also prone to autoPEEP (PEEP: positive end-expiratory pressure) due to obstructive disease and thus need little or no PEEP. Caution must be taken to avoid excessive elevated Paw to limit the risk of barotrauma and pneumothorax.

The restrictive lung disease patients will benefit from higher rates, lower $\mathrm{Vt}$, higher PEEP and I:E ratios closer to $1: 1$ or even 1.5:1 due to the poor compliance of the lungs.

The newly transplanted lung is going to behave much like the restrictive lung early in the post-transplant course which necessitates similar ventilator strategy for the immediate post lung transplant patient. It was also recognized that maintaining lower inspired oxygen concentration was beneficial for the newly transplanted lung. In the ARDS patient protective lung ventilation strategy for these patients keeping the $\mathrm{Vt}<6 \mathrm{~mL} / \mathrm{kg}$, RR of 14-18 breaths per minute, PEEP of $8-10 \mathrm{cmH}_{2} \mathrm{O}$ and $\mathrm{P}_{\text {plat }}$ $<30 \mathrm{cmH}_{2} \mathrm{O}$ has been shown to have a lower mortality (37). Maintaining a $\mathrm{P}_{\text {insp }}<20 \mathrm{cmH}_{2} \mathrm{O}$ and permissive hypercapnia is also typically advocated (38). Pressure controlled ventilation (PCV) may offer benefits for these patients 
as they are successful in lower both peak and plateau airway pressures. In a comparative study between pressure controlled and volume controlled ventilation, the mode of ventilation did not influence outcome as long as the PIP (in $\mathrm{PCV}$ ) or $\mathrm{P}_{\text {plat }}$ (in VCV) was maintained $<25 \mathrm{cmH}_{2} \mathrm{O}$ (39).

Utilization of PEEP has also shown to improve arterial oxygenation, functional residual capacity (FRC), decrease dead-space ventilation but this seems to have the most benefit when used with recruitment maneuvers. Absence of recruitment did not show the same amount of benefit (37). A number of other studies have called the utility of PEEP into question, so while PEEP may offer potential benefit in the lung transplant population, there does not appear to be solid evidence to support it (40). Recruitment maneuvers have also shown to improve A-a gradient, compliance, decreased level of inflammatory markers as well as decreased cumulative ventilator time. However, there is no standardized method to apply recruitment. This may be done in a stepwise fashion watching for improved physiologic parameters or by observing the lungs directly. It is important to note that recruitment measures should be followed immediately with application or reapplication of PEEP to maintain the benefit achieved (38).

ECMO has emerged as supportive therapy not only in the operative environment but also as a method of resting the lungs to allow time for the transplanted organs to recover from ischemia-reperfusion injury (41).

Ventilatory management of the donor appears to also be of potential benefit for better organ availability. Mascia et al. showed that, when compared to the consensus ventilator management of brain injured, brain dead patients, applying a protective ventilation strategy to the donor resulted in an increase in donor organs being available (42).

\section{Invasive monitoring}

Despite over 260 lung transplant centers conducting over 4,000 lung transplants annually (43), there are no formalized clinical guidelines for advanced intraoperative monitoring (44). Invasive arterial pressure (IAP) monitoring, pulmonary arterial catheter (PAC), central venous pressure (CVP), and transesophageal echocardiography (TEE) are widely accepted as essential tools for intraoperative monitoring in lung transplantation $(45,46)$. A recent survey of 176 transplant centers conducted by Tomasi et al. concluded that such monitoring techniques were employed nearly universally in all respondents (46). As such, pertinent literature regarding IAP, PAC, CVP and TEE will be discussed.

Pre-induction placement of a radial arterial line is a common practice that facilitates real-time hemodynamic monitoring during induction of anesthesia. Center specific protocols may call for the placement of a post-induction femoral arterial line as a means of monitoring central arterial pressures while also providing vascular access should the need for peripheral extra corporeal life support (ECLS) cannulation arise. PAC with continuous cardiac output or bolus thermodilution may be placed before or after induction of anesthesia. Placement of PAC before induction of anesthesia in patients with severe PHTN and right ventricular failure allows for precise management of preload and afterload during induction of anesthesia (47). However, post-induction placement of PAC minimizes anxiety, the need for premedication and increases in pulmonary vascular resistance (PVR) (48). The decision to place a PAC pre- or post-induction should be individualized based on the clinical context. CVP monitoring has been studied in correlation with postoperative mechanical ventilation requirements and morbidity. Maintaining CVP $<7 \mathrm{mmHg}$ may improve outcomes as reductions in fluid administration can improve pulmonary function (49). However, the context of such evidence must be considered when trying to apply to more contemporary practices. Many centers routinely utilize ECLS to facilitate lung transplantation and such fluid restrictive practices are not feasible. Optimal CVP during lung transplantation utilizing ECLS has not been adequately investigated to date. TEE provides many intraoperative benefits including guiding ECLS line and cannula placement and diagnosing acute intraoperative hemodynamic instability. A thorough preintervention TEE evaluation should be performed to include assessment of pulmonary veins (PV) and pulmonary arteries (PA). Evaluation of the left PA may be obstructed by ipsilateral mainstem bronchus (50). A prompt and accurate diagnosis for ventricular dysfunction, valvular dysfunction, hypovolemia, presence of patent foramen ovale, and right ventricular outflow obstruction can be gained with efficient TEE use (44,50). Although no formal guidelines have been established, TEE is essential for intraoperative evaluation of PV and artery stenosis (51). Standard two dimensional (2D), color Doppler, and spectral Doppler techniques can be utilized for evaluation of PV and PA stenosis. PV stenosis should be suspected in patients with PV diameter $<0.5 \mathrm{~cm}$, turbulent PV flow, PV peak systolic flow velocity $>1 \mathrm{~m} / \mathrm{s}$, and PV-left atrial pressure gradient $10-12 \mathrm{mmHg}$ $(44,50,52)$. It is important to note that graft failure is highly 
associated with $\mathrm{PV}$ diameter $<2.5 \mathrm{~mm}$ and velocity $>1.6 \mathrm{~m} / \mathrm{s}$ $(44,50)$. Clinical consensus for evaluation of PA stenosis is difficult to achieve due to insufficient data. Recent recommendations include laminar flow with Color Doppler and anastomosis should be no less than $75 \%$ diameter of donor's ipsilateral PA (50).

\section{Hemodynamic management}

The management of the lung transplant patient's hemodynamic status involves management of the inotropic support as well as management of the systemic and PVR.

Endothelial relaxing factor that was eventually determined to be nitric oxide (NO) plays a key role in being able to manage both the PVR and the areas of ventilation-perfusion (V/Q) mismatch in the patient with ischemia-reperfusion injury (53). Animal studies had also suggested that NO had properties that could potentially be protective against reperfusion injury but this has not been demonstrated to improve outcomes despite improving oxygenation and the effects of NO on PVR $(54,55)$.

Prior to the development of widespread availability of inhaled NO, prostaglandins (PGE) were the only other pharmacologic alternatives to being able to manage the load on the right ventricle in patients who had concomitant PHTN. They had been shown in animal models to improve outcomes after lung transplantation (56). The main disadvantage of intravenous prostaglandins was that it has a relatively long half-life which leads to systemic hypotension that may not be desirable in the patient with a tenuous right ventricle that relies on good coronary perfusion pressure. The ability to easily deliver $\mathrm{NO}$ in a precise fraction via the ventilator circuit has made it a mainstay for managing the lung transplant patient intraoperatively and into the ICU. Inhaled prostaglandin (epoprostenol) has a shorter half-life and therefore less effect on the SVR (57). It can be delivered in an aerosolized form and is much cheaper to use that $\mathrm{NO}$ (57-59).

The delivery system which entails $8 \mathrm{~mL} / \mathrm{hr}$ delivery via an ultrasonic nebulizer (58), however, can to be cumbersome to use. One major factor that works against more widespread use of NO is the cost of delivery. Some centers have who previously used NO have returned to using inhaled prostaglandins and milrinone in an attempt to provide the therapy without the significant cost inherent to NO delivery.

Milrinone, a phospho-diesterase-3 inhibitor has been shown previously to attenuate lung injury in the acute lung injury model (60) and has been shown to be of benefit in heart transplant patients is starting to be used in place of $\mathrm{NO}$ and PGE although the data for its widespread use is sparse but trials are ongoing.

Early hemodynamic collapse has been demonstrated to be an independent risk factor for mortality and thus needs to be treated promptly with inotropes (61). Inotropic support strategy should be determined based on the patients underlying baseline cardiac function, in particular the right ventricular function in patients with elevated pulmonary pressures, and degree of vasodilation. Dopamine, epinephrine and norepinephrine are widely used as are dobutamine and milrinone (62). Vasopressin may be chosen in the setting of needing an increase in SVR without elevating the PVR to optimize RV perfusion without increasing afterload (39).

\section{Special considerations}

The perioperative and anesthetic management of lung transplant patients are considerably impacted by the patient's underlying etiology. Although there is a lack of consensus guidelines on anesthetic management for lung transplantation, an emerging theme in the literature is that clinical care should be driven by the patient's underlying etiology (62). Pulmonary pathology that warrants lung transplantation can be divided into four common categories-obstructive, restrictive, suppurative, and PHTN (63). While, a recent review by Martin et al. (Table 1) provides an in-depth analysis on the impact of presenting disease on lung transplantation, we will focus on the special concerns for patients with COPD, cystic fibrosis (CF) and PHTN.

COPD was once the leading indication for lung transplantation in the last decade it is now the second most common indication (63). Mortality in COPD has been linked to comorbid conditions such as cardiovascular disease and cancer often resulting in more deaths than respiratory failure (64). As such, perioperative evaluation and testing is essential to exclude those with comorbid conditions and improve outcomes. In addition, assessment of preoperative hypercapnia and hypoxia should be obtained to stratify risk of induction of anesthesia. Induction of anesthesia should be conducted to maximize pre-oxygenation and minimize development of atelectasis and continuous positive airway pressure (CPAP) may be used to assist in accomplishing these goals $(65,66)$. Intraoperative anesthetic management focuses on combating the physiologic effects of air trapping 
Table 1 Key points for the perioperative anesthetic management of lung transplantation based on presenting disease etiology

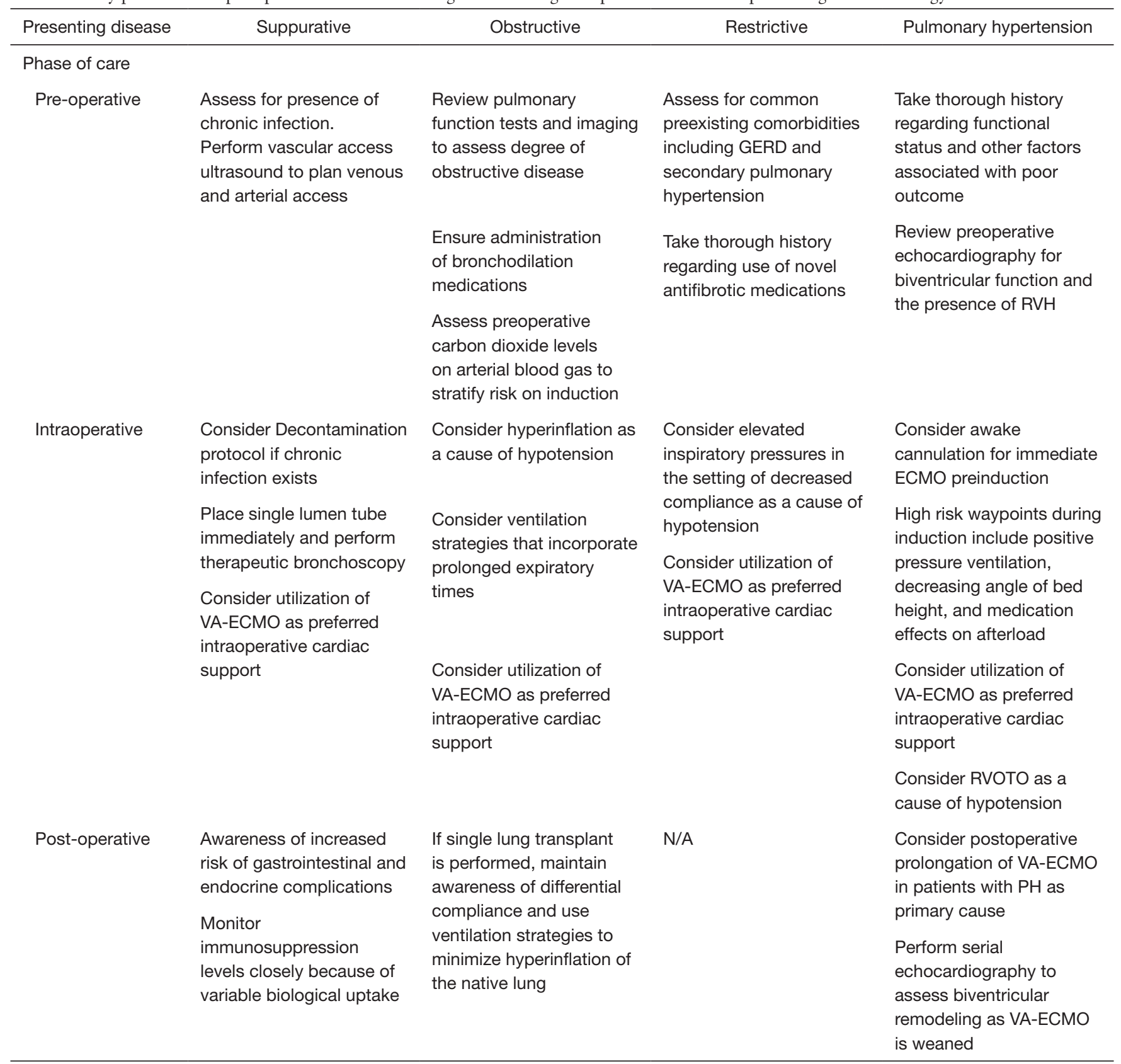

Ref: Martin et al. (62) Permissions obtained. VA-ECMO, veno-arterial extracorporeal membrane oxygenation.

and lung hyperinflation caused by COPD. Ventilation strategies to minimize this effect include reducing tidal volumes, reducing RRs, and increasing expiratory time (I:E ratios of $1: 3$ or more) $(62,65)$. This practice frequently results in permissive hypercapnia in an effort to minimize pulmonary barotrauma. It is important to prevent the development of intrinsic positive end-expiratory pressure also known as "breath stacking" which can lead to increased intrathoracic pressure, decreased systemic venous return, hypotension, and in some instances increased PVR leading to right heart strain (65). Perhaps the most difficult intraoperative task is maintaining oxygenation and adequate gas exchange during OLV. OLV increases the degree of pulmonary shunt which can lead to increased hypoxemia 
and hemodynamic instability. Inotropic agents, vasoactive agents, and inhaled nitric oxide function as adjuncts in this situation (67). If pharmacologic agents fail to stabilize hemodynamics, surgical clamping of the PA may improve the pulmonary shunt while simultaneously increasing right ventricular afterload (1). In the event of continued instability, ECMO initiation should be considered (52). In the event single lung transplantation is performed, special attention should be made to the differing of lung compliance between the native and donor lung in order to avoid dynamic hyperinflation (62).

CF continues to decrease slowly as an underlying etiology for lung transplantation, totaling only $15.4 \%$ $(\mathrm{n}=8,958)$ of all lung transplants conducted now $(62,63)$. However, CF transplant patients continue to demonstrate the highest survival rates at a median of 9.5 years $(62,63)$. A study by Kerem et al. shows a $70 \%$ two year mortality when CF patient's forced expiratory volume-one second $\left(\mathrm{FEV}_{1}\right)$ falls below $20 \%$ of predicted value $(68)$. As such patients should be considered for lung transplant when their $\mathrm{FEV}_{1}$ falls below 30\% (69). Chronic bacterial colonization of the airway is common in CF patients. Preoperative evaluation for multidrug resistant organisms should occur and organisms such as Burkbolderia cenocepacia and Mycobacteriums abscessus should raise concern for transplantation. Burkbolderia cenocepacia is the causative agent for necrotizing pneumonia leading to sepsis and death; it carries the highest risk for morbidity and mortality of all CF microorganisms (70). Mycobacteriums abscessus is difficult to treat and some transplant centers show higher mortality with chronic infection (70). As a result, most transplant centers consider infection with these organisms a strong relative or absolute contraindication to transplant (65). Ventilation of CF patients is complicated by profuse and copious secretions and clearance of these secretions should be attempted early to maximize value (69). CF patients have increased risk for prior thoracic procedures due to recurrent pneumothoraces and the prognosis is poor after first pneumothorax occurrence, with a median survival of 2.5 years (69). While the frequency of perioperative bleeding is significantly higher in patients with prior intrathoracic procedures and pleurodesis, the outcomes are comparable in experienced centers $(69,71)$.

Idiopathic pulmonary arterial hypertension (IPAH) is one of the least frequent etiologies for lung transplant with only 1,921 patients transplanted over the past 26 years (63). According to the International Society for Heart and Lung Transplantation Registry data, the median survival for IPAH is 6.3 years (63), yet has the highest three year mortality rates at $30 \%(72,73)$. Preoperative evaluation of these patients should involve a thorough investigation of the patient's functional status, right and left ventricular function, and other comorbidities (74). Perioperative factors associated with elevated mortality in IPAH lung transplantation specifically include syncope, hyponatremia, and decreased ventricular function (62). A well-planned anesthetic induction should include a plan to mitigate the initial pharmacological induced decrease of preload, decrease afterload, apnea, and increased thoracic pressure secondary to positive pressure ventilation (62). The use of inotropic agents to aid in severe right ventricular dysfunction in the setting of increased diuresis and decreased preload should be used judiciously so as not to cause right ventricular outflow obstruction (62). Recently, several organizations have transitioned to planned or prophylactic ECMO for IPAH patients (75). Favorable outcomes have been shown with the use of planned intraoperative and prolonged ECMO for IPAH, allowing the left and right ventricle to remodel $(62,73)$. Multiples studies showed improved survival rates at 1 year over $90 \%(73,75)$.

\section{Anesthetic management of the patient with severe PHTN}

PHTN is one of the most difficult aspects of ESLD to manage in the perioperative period. Challenges in anesthetic management arise from both end-stage cardiac and pulmonary dysfunction, and any interventional approach should revolve around the principle of the integration of heart and lungs as a functional cardiopulmonary unit (76).

Preoperative approach of these patients should include a thorough history and physical, as both subjective and objective measures of patient disease to include selfreported functional status, echocardiography, and right heart catheterization have been shown to provide valuable information regarding the severity of PHTN within the perioperative period (76-78). Immediate challenges prior to induction may include patient inability to lay flat, high oxygenation supplementation requirements, potential difficult airway, and right ventricular failure. While timing of the application of invasive monitoring such as PACs has been debated within the literature, the use of standard ASA monitors and an awake arterial line for hemodynamic monitoring are highly recommended (48).

Physiological goals for induction should focus on ameliorating negative consequences within both the cardiac and pulmonary systems. From the cardiac perspective, 
avoidance of dysrhythmias, increases in right ventricular afterload, right ventricular end-diastolic pressure, and decreases of coronary perfusion pressure are vital. From the pulmonary perspective, rapid acquisition of the airway in the hopes of avoiding hypoxemia or hypercapnia is recommended. Additionally, the use of positive pressure ventilation, which may negatively affect preload conditions within the cardiopulmonary unit, should be tailored to balance appropriate minute ventilation and peak airway pressures with stable hemodynamics (47).

The use of intraoperative ECLS support is highly recommended for PHTN patients and may be either VAECMO or CPB. In high-risk patients, pre-induction groin sheath cannulation may be considered, with planned ECLS being VA-ECMO, CPB, or a hybrid VA-ECMO/ $\mathrm{CPB}$ circuit to allow for ease of intraoperative conversion $(79,80)$. Once induction is achieved, the use of ECLS may proceed with a variety of configurations, including peripheral or central, depending on preference of the operative team (81). Due to chronic under-filling of the left ventricle, as well as acute remodeling of the right ventricle, recommendations from high performing centers includes the elective postoperative continuation of VA-ECMO (72). In conclusion, PHTN is one of the most challenging physiologic processes associated with ESLD, and a complete perioperative approach tailored to the patient's underlying condition is recommended for optimal success.

\section{Management of extracorporeal circulation in lung transplantation}

The perioperative management of lung transplantation impacts outcomes and has been shown to vary considerably between medical centers across the globe $(39,52)$. A key aspect of this intraoperative management is the use of ECLS. Lung transplantation can be performed with or without the use of ECLS, and the use of ECLS can occur during the preoperative, intraoperative, or postoperative phases of care.

The types of ECLS include CPB, VV-ECMO, and VAECMO (82,83). A strategic approach for selection of ECLS type depends both on the perioperative phase of care as well as underlying etiology of lung disease $(14,62)$.

\section{Preoperative use of ECLS}

The use of ECLS as a preoperative bridge has increased significantly during the most recent era of lung transplantation with improving patient safety and outcomes (84). Preoperative bridging with ECLS can be achieved by the use of either VV-ECMO or VA-ECMO. VV-ECMO is predominately used in isolated pulmonary failure while VA-ECMO is utilized in the setting of both cardiac and pulmonary failure (85). Contraindications for the use of ECMO bridging can be either absolute or relative, with absolute including sepsis, metastatic cancer, acute neurological dysfunction, and multiple organ failure (85). Relative contraindications include advanced age, obesity, or prolonged use of mechanical ventilation (85).

Hashimoto et al. recently reported their experience with VV-ECMO bridging for lung transplantation and outcomes (86). The retrospective cohort study included 34 patients, and the reported median duration of bridging was 12 days. They concluded that the use of bridging VV-ECMO during the intraoperative phase had similar outcomes for patients as compared to VA-ECMO, and that continuation of VV-ECMO bridging for intraoperative management was reasonable strategy.

In 2018, Hakim et al. reported outcomes of ECMO as a bridge to lung transplantation. While the predominant cohort of patients was bridged with dual-lumen single cannulas (43\%), $20 \%$ of the patients were bridged with VAECMO. Outcomes were assessed, and 30-day, 1- and 3-year survivals were $92 \%, 85 \%$, and $80 \%$. Based on their study, the authors note that favorable perioperative outcomes can be achieved with early bridging intervention in the appropriate patient population (87).

\section{Intraoperative use of ECLS}

The use of intraoperative ECLS can be either planned or emergent. Mohite et al. recently noted that emergent use of $\mathrm{CPB}$ within lung transplantation is associated with decreased survival at 1-, 2-, and 3-year intervals as compared to either no $\mathrm{CPB}$ or planned $\mathrm{CPB}$ strategies $(\mathrm{P}<0.001)$. When ECLS is planned, an approach of either $\mathrm{CPB}$ or VA-ECMO is generally considered (83).

Traditionally, CPB has been the preferred method of intraoperative ECLS support. However, recent trends show the emergence of VA-ECMO as the preferred method of intraoperative ECLS (75). Data show the use of CPB being associated with increased risk of PGD (88) while the use of VA-ECMO has been associated with improved rates of survival as compared to no ECLS at all (75). The largest study to date examining the use of VA-ECMO versus no ECLS was produced by the Vienna group, who reported 
statistically significant data for patient survival at 1-, 3-, and 5 -year $(\mathrm{P}=0.04)(75)$. The working theory put forward by the group in their 2018 paper is a consideration for attenuation of the ischemic-reperfusion injury by diverting the majority of cardiac output away from the lung transplant graft. Preferred intraoperative cannulation is central via a clamshell incision, with peripheral VA-ECMO placed for post-operative prolongation in unstable patients. Some groups have nearly abandoned CPB entirely in favor of ECMO (89).

\section{Postoperative use of ECLS}

While data regarding institution of ECLS in the postoperative setting are limited, it is most often employed due to the deleterious effects of developing PGD (82). Data regarding postoperative ECLS outcomes show that, much like preoperative bridging, early intervention can be associated with improved outcomes (82).

\section{Pain management strategies in lung transplantation}

Management of acute post-operative pain the lung transplant recipient can be challenging, yet a balanced approach that is mindful of optimization cardiopulmonary function while minimizing side effects can achieve a positive impact on outcomes $(52,90)$. Not only is acute post-operative pain a challenge, but chronic pain is as well. A recent survey of lung transplantation patients reported an incidence of chronic pain of $51 \% 1$ year post initial surgery (91).

Given these circumstances, techniques involving the use of regional anesthetics are often employed. The most frequently studied, thoracic epidural analgesia (TEA), is noted in literature to be the "gold standard" (52). Overall, data regarding the impact of analgesic approach on lung transplantation outcomes are limited, however, TEA has been shown to decrease postoperative mechanical ventilation time in these patients (92). Other pain management strategies reported include the use of paravertebral catheters, serratus anterior plane block, and erector spinae block $(52,93)$. Larger studies are necessary to delineate the optimal pain management approach in this patient population, and should include direct comparisons of specific regional anesthetic techniques to each other.

\section{Conclusions}

The practice of lung transplantation has expanded and evolved significantly over the past 37 years since that first success for Cooper. This has required changes in the practice of anesthesiology. Much is still to be learned about what effect, if any, particular anesthetic agents might have on the transplanted organ and recipient. It is unknown if anesthetic agents have any benefit to the donor organ in the Ex Vivo Lung Perfusion (EVLP) phase of organ recovery and possible rehabilitation prior to implantation.

Greater use of ECMO to allow an unintubated patient to rehab in preparation for their lung transplant has the potential to bring patients to their surgery in better physiological shape (39). The use $\mathrm{CO}_{2}$ devices like Novalung and Decap allow to sustain patients when ventilation is not effective enough to manage patients' severe hypercapnia in the presence of only mild to moderate hypoxemia.

Newer less-invasive methods to monitor hemodynamics may be developed to lessen the stress on the recipient as well as the risk for infection that current techniques present by virtue of their invasive nature.

We continue to learn about the potentially deleterious effects of mechanical ventilation and whether there are approaches that can be employed to lessen the effect on the newly transplanted organs.

Echocardiography is a very valuable tool to guide both the hemodynamic care of the patient as well evaluation of the surgical procedure and may develop further as a more reliable method of monitoring patient's hemodynamic status less invasively.

\section{Acknowledgments}

Funding: None.

\section{Footnote}

Provenance and Peer Review: This article was commissioned by the Guest Editor (Jonathan D'Cunha) for the series "Lung Transplantation: Past, Present, and Future" published in Fournal of Thoracic Disease. The article has undergone external peer review.

Conflicts of Interest: All authors have completed the ICMJE uniform disclosure form (available at http:// dx.doi.org/10.21037/jtd-2021-10). The series "Lung Transplantation: Past, Present, and Future" was commissioned by the editorial office without any funding sponsorship. The authors have no other conflicts of interest 
to declare.

Ethical Statement: All authors are accountable for all aspects of the work in ensuring that questions related to the accuracy or integrity of any part of the work are appropriately investigated and resolved.

Open Access Statement: This is an Open Access article distributed in accordance with the Creative Commons Attribution-NonCommercial-NoDerivs 4.0 International License (CC BY-NC-ND 4.0), which permits the noncommercial replication and distribution of the article with the strict proviso that no changes or edits are made and the original work is properly cited (including links to both the formal publication through the relevant DOI and the license). See: https://creativecommons.org/licenses/by-nc-nd/4.0/.

\section{References}

1. Baez B, Castillo M. Anesthetic considerations for lung transplantation. Semin Cardiothorac Vasc Anesth 2008;12:122-7.

2. Aris R, Barbers RG, Barst R, et al. International guidelines for the selection of lung transplant candidates. Am J Respir Crit Care Med 1998;158:335-9.

3. Orens JB, Estenne M, Arcasoy S, et al. International Guidelines for the Selection of Lung Transplant Candidates: 2006 Update-A Consensus Report From the Pulmonary Scientific Council of the International Society for Heart and Lung Transplantation. J Heart Lung Transplant 2006;25:745-55.

4. Weill D, Benden C, Corris PA, et al. A consensus document for the selection of lung transplant candidates: 2014-An update from the Pulmonary Transplantation Council of the International Society for Heart and Lung Transplantation. J Heart Lung Transplant 2015;34:1-15.

5. Verleden GM, Dupont L, Yserbyt J, et al. Recipient selection process and listing for lung transplantation. J Thorac Dis 2017;9:3372-84.

6. Pinho DF, Banga A, Torres F, et al. Ventilation perfusion pulmonary scintigraphy in the evaluation of pre-and post-lung transplant patients. Transplant Rev (Orlando) 2019;33:107-14.

7. Zanotti G, Hartwig MG, Castleberry AW, et al. Preoperative mild-to-moderate coronary artery disease does not affect long-term outcomes of lung transplantation. Transplantation 2014;97:1079-85.

8. Koprivanac M, Budev MM, Yun JJ, et al. How important is coronary artery disease when considering lung transplant candidates? J Heart Lung Transplant 2016;35:1453-61.

9. Seoane L, Arcement LM, Valentine VG, et al. Longterm survival in lung transplant recipients after successful preoperative coronary revascularization. J Thorac Cardiovasc Surg 2005;130:538-41.

10. Yerasi C, Roy SB, Olson M, et al. Outcomes of lung transplant recipients with preoperative atrial fibrillation. Asian Cardiovasc Thorac Ann 2018;26:127-32.

11. Porteous MK, Ky B, Kirkpatrick JN, et al. Diastolic Dysfunction Increases the Risk of Primary Graft Dysfunction after Lung Transplant. Am J Respir Crit Care Med 2016;193:1392-400.

12. Diamond JM, Lee JC, Kawut SM, et al. Clinical risk factors for primary graft dysfunction after lung transplantation. Am J Respir Crit Care Med 2013;187:527-34.

13. Shah PR, Boisen ML, Winger DG, et al. Extracorporeal Support During Bilateral Sequential Lung Transplantation in Patients With Pulmonary Hypertension: Risk Factors and Outcomes. J Cardiothorac Vasc Anesth 2017;31:418-25.

14. Ius F, Tudorache I, Warnecke G. Extracorporeal support, during and after lung transplantation: the history of an idea. J Thorac Dis 2018;10:5131-48.

15. Grimm JC, Lui C, Kilic A, et al. A Risk Score to Predict Acute Renal Failure in Adult Patients After Lung Transplantation. Ann Thorac Surg 2015;99:251-7.

16. Grimm JC, Valero V III, Kilic A, et al. Preoperative Performance Status Impacts Perioperative Morbidity and Mortality After Lung Transplantation. Ann Thorac Surg 2015;99:482-9.

17. Osho A, Mulvihill M, Lamba N, et al. Is Functional Independence Associated With Improved Long-Term Survival After Lung Transplantation? Ann Thorac Surg 2018;106:79-84.

18. Castleberry AW, Englum BR, Snyder LD, et al. The Utility of Preoperative Six-Minute-Walk Distance in Lung Transplantation. Am J Respir Crit Care Med 2015;192:843-52.

19. Spruit MA, Singh SJ, Garvey C, et al. An official American Thoracic Society/European Respiratory Society statement: key concepts and advances in pulmonary rehabilitation. Am J Respir Crit Care Med 2013;188:e13-e64.

20. Hoffman M, Chaves G, Ribeiro-Samora GA, et al. Effects of pulmonary rehabilitation in lung transplant candidates: a systematic review. BMJ Open 2017;7:e013445.

21. Pehlivan E, Balcı A, Kılıç L, et al. Preoperative Pulmonary Rehabilitation for Lung Transplant: Effects on Pulmonary Function, Exercise Capacity, and Quality of Life; First 
Results in Turkey. Exp Clin Transplant 2018;16:455-60.

22. Langer D. Rehabilitation in Patients before and after Lung Transplantation. Respiration 2015;89:353-62.

23. Bottiger BA, Nicoara A, Snyder LD, et al. Frailty in the End-Stage Lung Disease or Heart Failure Patient: Implications for the Perioperative Transplant Clinician. J Cardiothorac Vasc Anesth 2019;33:1382-92.

24. Singer JP, Diamond JM, Gries CJ, et al. Frailty Phenotypes, Disability, and Outcomes in Adult Candidates for Lung Transplantation. Am J Respir Crit Care Med 2015;192:1325-34.

25. Rozenberg D, Mathur S, Wickerson L, et al. Frailty and Clinical Benefits with Lung Transplantation. J Heart Lung Transplant 2018;37:1245-53.

26. Singer JP, Diamond JM, Anderson MR, et al. Frailty phenotypes and mortality after lung transplantation: A prospective cohort study. Am J Transplant 2018;18:19952004.

27. Lederer DJ, Kawut SM, Wickersham N, et al. Obesity and primary graft dysfunction after lung transplantation: the Lung Transplant Outcomes Group Obesity Study. Am J Respir Crit Care Med 2011;184:1055-61.

28. Fernandez R, Safaeinili N, Kurihara C, et al. Association of body mass index with lung transplantation survival in the United States following implementation of the lung allocation score. J Thorac Cardiovasc Surg 2018;155:18719.e1873.

29. Kim CY, Kim BK, Kim SY, et al. Usefulness of the preoperative prognostic nutritional index score as a predictor of the outcomes of lung transplantation: A single-institution experience. Clin Nutr 2019;38:2423-9.

30. Kelm DJ, Bonnes SL, Jensen MD, et al. Pre-transplant wasting (as measured by muscle index) is a novel prognostic indicator in lung transplantation. Clin Transplant 2016;30:247-55.

31. Singer JP, Peterson ER, Snyder ME, et al. Body Composition and Mortality after Adult Lung Transplantation in the United States. Am J Respir Crit Care Med 2014;190:1012-21.

32. Jomphe V, Mailhot G, Damphousse V, et al. The Impact of Waiting List BMI Changes on the Short-term Outcomes of Lung Transplantation. Transplantation 2018;102:318-25.

33. Anderson MR, Udupa JK, Edwin E, et al. Adipose tissue quantification and primary graft dysfunction after lung transplantation: The Lung Transplant Body Composition study. J Heart Lung Transplant 2019;38:1246-56.

34. Tinckam KJ, Keshavjee S, Chaparro C, et al. Survival in sensitized lung transplant recipients with perioperative desensitization. Am J Transplant 2015;15:417-26.

35. Rolly G, Malcolm-Thomas B, Verschraegen R, et al. Anesthesia during human lung transplantation and early postoperative respiratory treatment. Int Anesthesiol Clin 1972;10:79-92.

36. Barnes L, Reed RM, Parekh KR, et al. Mechanical ventilation for the lung transplant recipient. Curr Pulmonol Rep 2015;4:88-96.

37. Maisch S, Reissmann H, Fuellekrug B, et al. Compliance and Dead Space Fraction Indicate an Optimal Level of Positive End-Expiratory Pressure After Recruitment in Anesthetized Patients. Anesth Analg 2008;106:175-81.

38. Lucangelo U, Del Sorbo L, Boffini M, et al. Protective ventilation for lung transplantation. Curr Opin Anaesthesiol 2012;25:170-4.

39. Gelzinis TA. Anesthetic Management of Lung Transplantation: Center Specific Practices and Geographical and Centers Size Differences. J Cardiothorac Vasc Anesth 2018;32:70-2.

40. Verbeek GL, Myles PS. Intraoperative protective ventilation strategies in lung transplantation. Transplant Rev (Orlando) 2013;27:30-5.

41. Turner DA, Ofori-Amanfo G, Williford WL, et al. Lung protective ventilation: a summary of the current evidence from the 2012 American Association for Respiratory Care International Congress. Expert Rev Respir Med 2013;7:209-12.

42. Mascia L, Pasero D, Slutsky AS, et al. Effect of a Lung Protective Strategy for Organ Donors on Eligibility and Availability of Lungs for Transplantation: A Randomized Controlled Trial. JAMA 2010;304:2620-7.

43. Chambers DC, Cherikh WS, Harhay MO, et al. The International Thoracic Organ Transplant Registry of the International Society for Heart and Lung Transplantation: Thirty-sixth adult lung and heart-lung transplantation Report-2019; Focus theme: Donor and recipient size match. J Heart Lung Transplant 2019;38:1042-55.

44. Kachulis B, Mitrev L, Jordan D. Intraoperative anesthetic management of lung transplantation patients. Best Pract Res Clin Anaesthesiol 2017;31:261-72.

45. Della Rocca G, Brondani A, Costa MG. Intraoperative hemodynamic monitoring during organ transplantation: what is new? Curr Opin Organ Transplant 2009;14:291-6.

46. Tomasi R, Betz D, Schlager S, et al. Intraoperative Anesthetic Management of Lung Transplantation: CenterSpecific Practices and Geographic and Centers Size Differences. J Cardiothorac Vasc Anesth 2018;32:62-9.

47. Hargrave J. Con: Preinduction Pulmonary Artery Catheter 
Placement Is Advisable in Patients With Right Ventricular Dysfunction Secondary to Severe Pulmonary Hypertension. J Cardiothorac Vasc Anesth 2017;31:1514-8.

48. Au S, Nguyen L, Banks D. Pro: Pulmonary Artery Catheter Placement After Anesthetic Induction in Patients Undergoing Pulmonary Thromboendarterectomy. J Cardiothorac Vasc Anesth 2017;31:1511-3.

49. Pilcher DV, Scheinkestel CD, Snell GI, et al. High central venous pressure is associated with prolonged mechanical ventilation and increased mortality after lung transplantation. J Thorac Cardiovasc Surg 2005;129:912-8.

50. Abrams BA, Melnyk V, Allen WL, et al. TEE for Lung Transplantation: A Case Series and Discussion of Vascular Complications. J Cardiothorac Vasc Anesth 2020;34:733-40.

51. González-Fernández C, González-Castro A, RodríguezBorregán JC, et al. Pulmonary venous obstruction after lung transplantation. Diagnostic advantages of transesophageal echocardiography. Clin Transplant 2009;23:975-80.

52. Martin AK, Yalamuri SM, Wilkey BJ, et al. The Impact of Anesthetic Management on Perioperative Outcomes in Lung Transplantation. J Cardiothorac Vasc Anesth. 2020;34:1669-80.

53. Kemming GI, Merkel MJ, Schallerer A, et al. Inhaled nitric oxide (NO) for the treatment of early allograft failure after lung transplantation. Intensive Care Med 1998;24:1173-80.

54. Meade MO, Granton JT, Matte-Martyn A, et al. A Randomized Trial of Inhaled Nitric Oxide to Prevent Ischemia-Reperfusion Injury after Lung Transplantation. Am J Respir Crit Care Med 2003;167:1483-9.

55. Ardehali A, Laks H, Levine M, et al. A prospective trial of inhaled nitric oxide in clinical lung transplantation. Transplantation 2001;72:112-5.

56. Aoe M, Trachiotis GD, Okabayashi K, et al. Administration of prostaglandin E1 after lung transplantation improves early graft function. Ann Thorac Surg 1994;58:655-61.

57. Della Rocca G, Coccia C, Pompei L, et al. Inhaled aerosolized prostaglandin E1, pulmonary hemodynamics, and oxygenation during lung transplantation. Minerva Anestesiol 2008;74:627-33.

58. Slinger P. 2012. Anaesthetic management for lung transplantation. Pdfssemanticscholar.org.

59. Fiser SM, Cope JT, Kron IL, et al. Aerosolized prostacyclin (epoprostenol) as an alternative to inhaled nitric oxide for patients with reperfusion injury after lung transplantation. J Thorac Cardiovasc Surg 2001;121:981-2.

60. Bueltmann M, Kong X, Mertens M, et al. Inhaled milrinone attenuates experimental acute lung injury. Intensive Care Med 2009;35:171-8.

61. Thabut G, Vinatier I, Brugière $\mathrm{O}$, et al. Influence of preservation solution on early graft failure in clinical lung transplantation. Am J Respir Crit Care Med 2001;164:1204-8.

62. Martin AK, Fritz AV, Wilkey BJ. Anesthetic management of lung transplantation: impact of presenting disease. Curr Opin Anaesthesiol 2020;33:43-9.

63. Chambers DC, Cherikh WS, Goldfarb SB, et al. The International Thoracic Organ Transplant Registry of the International Society for Heart and Lung Transplantation: Thirty-fifth adult lung and heart-lung transplant report-2018; Focus theme: Multiorgan Transplantation. J Heart Lung Transplant 2018;37:1169-83.

64. Reed RM, Cabral HJ, Dransfield MT, et al. Survival of Lung Transplant Candidates With COPD: BODE Score Reconsidered. Chest 2018;153:697-701.

65. Duggappa DR, Rao GV, Kannan S. Anaesthesia for patient with chronic obstructive pulmonary disease. Indian J Anaesth 2015;59:574-83.

66. Khetarpal R, Bali K, Chatrath V, et al. Anesthetic considerations in the patients of chronic obstructive pulmonary disease undergoing laparoscopic surgeries. Anesth Essays Res 2016;10:7-12.

67. Myles PS. Lessons from lung transplantation for everyday thoracic anesthesia. Anesthesiol Clin North Am 2001;19:581-90, vii.

68. Kerem E, Reisman J, Corey M, et al. Prediction of mortality in patients with cystic fibrosis. N Engl J Med 1992;326:1187-91.

69. Lenihan M, Mullane D, Buggy D, et al. Anesthesia for Lung Transplantation in Cystic Fibrosis: Retrospective Review from the Irish National Transplantation Centre. J Cardiothorac Vasc Anesth 2018;32:2372-80.

70. Dupont L. Lung transplantation in cystic fibrosis patients with difficult to treat lung infections. Curr Opin Pulm Med 2017;23:574-9.

71. Meachery G, De Soyza A, Nicholson A, et al. Outcomes of lung transplantation for cystic fibrosis in a large UK cohort. Thorax 2008;63:725-31.

72. Moser B, Jaksch P, Taghavi S, et al. Lung transplantation for idiopathic pulmonary arterial hypertension on intraoperative and postoperatively prolonged extracorporeal membrane oxygenation provides optimally controlled reperfusion and excellent outcome. Eur J Cardiothorac Surg 2018;53:178-85.

73. Tudorache I, Sommer W, Kühn C, et al. Lung 
Transplantation for Severe Pulmonary HypertensionAwake Extracorporeal Membrane Oxygenation for Postoperative Left Ventricular Remodelling. Transplantation 2015;99:451-8.

74. McGlothlin D, Ivascu N, Heerdt PM. Anesthesia and pulmonary hypertension. Prog Cardiovasc Dis 2012;55:199-217.

75. Hoetzenecker K, Schwarz S, Muckenhuber M, et al. Intraoperative extracorporeal membrane oxygenation and the possibility of postoperative prolongation improve survival in bilateral lung transplantation. J Thorac Cardiovasc Surg 2018;155:2193-2206.e3.

76. Vonk Noordegraaf A, Chin KM, Haddad F, et al. Pathophysiology of the right ventricle and of the pulmonary circulation in pulmonary hypertension: an update. Eur Respir J 2019;53:1801900.

77. Shah AC, Ma K, Faraoni D, et al. Self-reported functional status predicts post-operative outcomes in non-cardiac surgery patients with pulmonary hypertension. PLoS One 2018;13:e201914.

78. Rosenkranz S, Preston IR. Right heart catheterisation: best practice and pitfalls in pulmonary hypertension. Eur Respir Rev 2015;24:642-52.

79. Martin AK, Harrison BA, Fritz AV, et al. Intraoperative management of a hybrid extracorporeal membrane oxygenation circuit for lung transplantation. J Card Surg 2020;35:3560-3.

80. Thomas M, Martin AK, Allen WL, et al. Lung Transplantation Using a Hybrid Extracorporeal Membrane Oxygenation Circuit. ASAIO J 2020;66:e123-5.

81. Martin AK, Jayaraman AL, Nabzdyk CG, et al. Extracorporeal Membrane Oxygenation in Lung Transplantation: Analysis of Techniques and Outcomes. J Cardiothorac Vasc Anesth 2021;35:644-61.

82. Gulack BC, Hirji SA, Hartwig MG. Bridge to Lung Transplantation and Rescue Post-Transplant: the Expanding Role of Extracorporeal Membrane Oxygenation. J Thorac Dis 2014;6:1070-9.

83. Mohite PN, Sabashnikov A, Patil NP, et al. The role of cardiopulmonary bypass in lung transplantation. Clin

Cite this article as: Murray AW, Boisen ML, Fritz A, Renew JR, Martin AK. Anesthetic considerations in lung transplantation: past, present and future. J Thorac Dis 2021;13(11):6550-6563. doi: 10.21037/jtd-2021-10
Transplant 2016;30:202-9.

84. Hayanga JWA, Hayanga HK, Holmes SD, et al. Mechanical ventilation and extracorporeal membrane oxygenation as a bridge to lung transplantation: Closing the gap. J Heart Lung Transplant 2019;38:1104-11.

85. Loor G, Simpson L, Parulekar A. Bridging to lung transplantation with extracorporeal circulatory support: when or when not? J Thorac Dis 2017;9:3352-61.

86. Hashimoto K, Hoetzenecker K, Yeung JC, et al. Intraoperative extracorporeal support during lung transplantation in patients bridged with venovenous extracorporeal membrane oxygenation. J Heart Lung Transplant 2018;37:1418-24.

87. Hakim AH, Ahmad U, McCurry KR, et al. Contemporary Outcomes of Extracorporeal Membrane Oxygenation Used as Bridge to Lung Transplantation. Ann Thorac Surg 2018;106:192-8.

88. Porteous MK, Diamond JM, Christie JD. Primary graft dysfunction: lessons learned about the first $72 \mathrm{~h}$ after lung transplantation. Curr Opin Organ Transplant 2015;20:506-14.

89. Hoechter DJ, Dossow von V, Winter H, et al. The Munich Lung Transplant Group: Intraoperative Extracorporeal Circulation in Lung Transplantation. Thorac Cardiovasc Surg 2015;63:706-14.

90. Feltracco P, Barbieri S, Milevoj M, et al. Thoracic epidural analgesia in lung transplantation. Transplant Proc 2010;42:1265-9.

91. Forsberg A, Claëson M, Dahlman GB, et al Pain, fatigue and well-being one to five years after lung transplantation - a nationwide cross-sectional study. Scand J Caring Sci 2018;32:971-8.

92. McLean SR, Homeyer von P, Cheng A, et al. Assessing the Benefits of Preoperative Thoracic Epidural Placement for Lung Transplantation. J Cardiothorac Vasc Anesth 2018;32:2654-61.

93. Gelzinis TA. An Update on Postoperative Analgesia Following Lung Transplantation. J Cardiothorac Vasc Anesth 2018;32:2662-4. 